\title{
THERMODYNAMICS OF ISING FERROMAGNET STUDIED BY THE REAL-SPACE RENORMALIZATION GROUP METHOD
}

\author{
W. REICII
}

Institule of Physics, A. Mickiewicz University, Matejki 48/49, 60-769 Poznan, Poland

K. Piotrkowska

SISSA, Trieste, Italy

\section{U. Esposito and A. Caramico D'Auria}

Dipartimento di Scienze Fisiche, Universita di Napoli, Napoli, Italy

(Received January 15, 1993; revised version. June 3, 1993)

The critical exponents of the 3D Ising model were calculated in the approximation of the fourth-order cumulant expansion. Thermodynamic functions in the high-temperature range are obtained.

PACS numbers: 64.60.Ak, 75.40.Cx

\section{Introduction}

Investigation of phase transitions in the generalized Ising models has been so far performed using a few versions of the method based on different realizations of the real-space renormalization group (RSRG) [1]. Effectiveness of each realization could be checked only retrospectively. In this work, similarly as in Refs. [2] and [3], the Niemeijer and van Leeuwen (NvL) transformation was chosen for determination of critical exponents and thermodynamic functions for an Ising ferromagnet on a simple cubic lattice. The calculations were performed up to the fourth order of the cumulant expansion.

\section{Thermodynamics}

The applied NvL transformation was in the form

$$
\exp \mathcal{H}^{\prime}\left\{S^{\prime}\right\}=\sum_{\{S\}} \prod_{i^{\prime}} \frac{1}{2}\left[1+S_{i^{\prime}}^{\prime} \operatorname{sgn}\left(\sum_{n=1}^{8} S_{i^{\prime}}^{n}\right)\right] \exp \mathcal{H}\{S\}
$$


On splitting the Hamiltonian $\mathcal{H}\{S\}$ into the zeroth part $\mathcal{H}_{0}\{S\}$ and the perturbational part $\mathcal{V}\{S\}$, we can write the transformation (1) as

$$
\mathcal{H}^{\prime}\left\{S^{\prime}\right\}=E_{0}+\langle\mathcal{V}\rangle_{0}+\frac{1}{2 !}\left\langle\left(\mathcal{V}-\langle\mathcal{V}\rangle_{0}\right)^{2}\right\rangle_{0}+\frac{1}{3 !}\left\langle\left(\mathcal{V}-\langle\mathcal{V}\rangle_{0}\right)^{3}\right\rangle_{0}+\ldots
$$

where the average $\langle\ldots\rangle_{0}$ is defined by

$$
\langle A\rangle_{0}=\frac{\sum_{\{S\}} \prod_{i^{\prime}} \frac{1}{2}\left[1+S_{i^{\prime}}^{\prime} \operatorname{sgn}\left(\sum_{n=1}^{8} S_{i^{\prime}}^{n}\right)\right] \exp \mathcal{H}_{0}\{S\} A\{S\}}{\sum_{\{S\}} \prod_{i^{\prime}} \frac{1}{2}\left[1+S_{i^{\prime}}^{\prime} \operatorname{sgn}\left(\sum_{n=1}^{8} S_{i^{\prime}}^{n}\right)\right] \exp \mathcal{H}_{0}\{S\}} .
$$

In the calculations two coupling parameters were taken into account, namely, a magnetic field $H$ and the nearest neighbours coupling $K$. Table presents the values of the critical indices $\left(\nu_{\mathrm{HTSE}}=0.638, \eta_{\mathrm{HTSE}}=0.041[4]\right)$ obtained applying the standard method $[5,7,8]$.

\section{TABLE}

Critical eigenvalues and exponents for the sc lattice.

\begin{tabular}{c|c|c|c|c}
\hline \hline $\begin{array}{c}\text { Order } \\
\text { of perturbation }\end{array}$ & $\lambda_{T}$ & $\lambda_{H}$ & $\nu$ & $\eta$ \\
\hline 1 & 2.371 & 6.908 & 0.803 & -0.577 \\
2 & 2.371 & 6.458 & 0.803 & -0.382 \\
3 & 3.095 & 5.578 & 0.614 & 0.041 \\
4 & 3.095 & 5.468 & 0.614 & 0.098
\end{tabular}

According to the NvL method, free energy density can be expressed as a series including all constant terms of transformation (2) while other thermodynamic functions can be obtained as appropriate derivatives of the free energy $[1,6]$. This work presents the results of calculations of thermodynamic functions for positive values of $K$. For a fixed $y$ ( $y$ is the ratio between the magnetic field and coupling constant) we calculated the free energy $(F)$, internal energy $(U)$, specific heat $(c)$, magnetization $(m)$ and susceptibility $(s)$ for $K$ values increasing from zero to the maximum value for which the series used for free energy calculation are convergent. For $y=0$ we consider $0 \leq K \leq 0.25$ by a step of 0.01 . For $K=0.26$ we find the series divergent so we repeat the procedure with a step of 0.001 and then 0.0001 until finally the series is found divergent for $K=0.2529$. We proceed in the same way for other values of $y$. The results are presented in Figs. 1a-e.

\section{Final remarks}

This work was aimed at checking whether further terms of the cumulant expansion in the NvL method would improve the accuracy of results or not.

The studies of this effect were undertaken as there have been reports of thermodynamic functions whose first few terms of their expansion in series were convergent while the following significantly deteriorated the results $[9,10]$. 

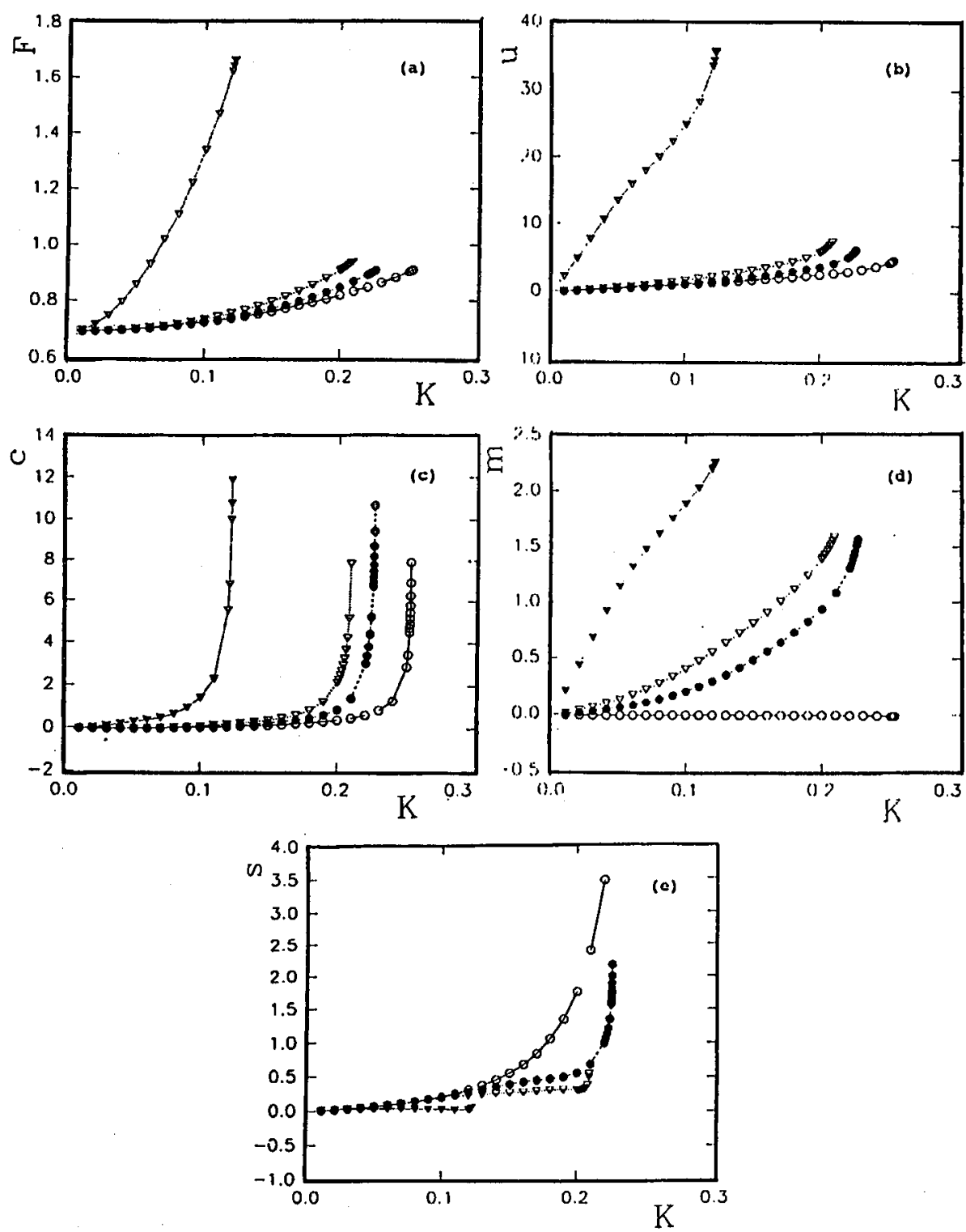

Fig. 1. Thermodynamic functions versus $K=J\left(k_{\mathrm{B}} T\right)^{-1}$; (a) free energy, (b) internal energy, (c) specific heat, (d) magnetization, (e) susceptibility; $\circ$ denotes $y=0, \bullet y=0.5$, $\nabla y=1$, full $\nabla y=10$.

The results of this work have proved the deterioration of results calculated including the fourth order of the expansion in series. As far as the critical exponents are concerned, the $\eta$ exponent in the fourth order gets more deviated from 
the value $\eta_{\text {HTSE }}$ than the corresponding value of the third order of the expansion. Moreover, the thermodynamic functions up to the fourth order of expansion could be obtained in a relatively narrower ligh-temperature range (low $K$ ) and we observed an unexpected and hard to explain change in convexity of the curves of magnetization $(m)$ and internal energy $(U)$ for high values of the $y$ parameter (high $H$ ).

\section{References}

[1] Real Space Renormalization, Eds. T.W. Burkhardt, J.M.J. van Leeuwen, Springer, Berlin 1982.

[2] W. Reich, H. Cofta, Phys. Status. Solidi B 134, K31 (1986).

[3] W. Reich, Phys. Status Solidi B 149, K163 (1988).

[4] M.A. Moore, D. Jasnow, M. Wortis, Phys. Rev. Lett. 22, 940 (1969).

[5] Th. Niemeijer, J.M.J. van Leeuwen, Physica 71, 17 (1974).

[6] L.S. Campana, A. Caramico D'Auria, U. Esposito, W. Reicl, Acla Magnelica VI, 3 (1989).

[7] W. Reich, J. Phys. A, Math. Gen. 17, 3553 (1984).

[8] T. Tatsumi, Prog. Theor. Phys. 59, 714 (1978).

[9] V.G. Vaks, A.I. Larkin, S.A. Pikin, Sov. Phys.-JETP 51, 361 (1966).

[10] Z. Onyszkiewicz, A. Wierzbicki, Phys. Lett. A 116, 335 (1986). 